\title{
Cranial Magnetic Resonance Spectroscopy: An Update of Metabolites and a Special Emphasis on Practical Points
}

\author{
Duzgun Yildirim¹, Onur Tutar², Deniz Alis², Gokhan Kuyumcu'2, Selim Bakan² \\ ${ }^{1}$ Centermed Advanced Imaging Center, Istanbul, Turkey \\ ${ }^{2}$ Depatment of Radiology, Cerrahpasa Medical Faculty, Istanbul University, Istanbul, Turkey \\ Email: yildirimduzgun@yahoo.com
}

Received 8 September 2014; revised 8 October 2014; accepted 7 November 2014

Copyright @ 2014 by authors and Scientific Research Publishing Inc.

This work is licensed under the Creative Commons Attribution International License (CC BY). http://creativecommons.org/licenses/by/4.0/

c) (i) Open Access

\begin{abstract}
Magnetic resonance spectroscopy (MRS) could be used for dozens of metabolites [1]. In this paper we will focus especially on proton (hydrogen) MRS. Generally published literatures consist of reviews and articles about metabolites are long, complicated and emphasise on physical or on technical data. But with this article, we will try to explain basically how MRS could be used in daily practice of radiologists. First of all we tried to search all current literature only referencing the main ones to make this review and we want all of our colleagues to give feedback about this review. With collection of all these trick points and line up to appropriate order, it is possible to make this review a common guide for radiologists and a useful tool while reporting MRS.
\end{abstract}

\section{Keywords}

Magnetic Resonance Spectroscopy, Analysis, Metabolite Markers, Radiology

\section{Introduction, Technique and Short Tips on Protocol}

After the 3 tesla MRS came into use, we are able to measure main metabolites more accurately, also with current softwares we are able to measure more metabolites. So with all those new developments, differential diagnosis of metabolical diseases, follow up of demyelinating diseases, differentiation of malignant and benign tumours and staging of tumours are much easier. The most important part of MRS is, it has ability to measure chemical component of the area that we study, non invasively (Figure 1).

Generally metabolites are distributed between water and lipid peaks. There are different techniques to obtain metabolite information but this is not a subject for this review. But at least we can say that there are mainly two 


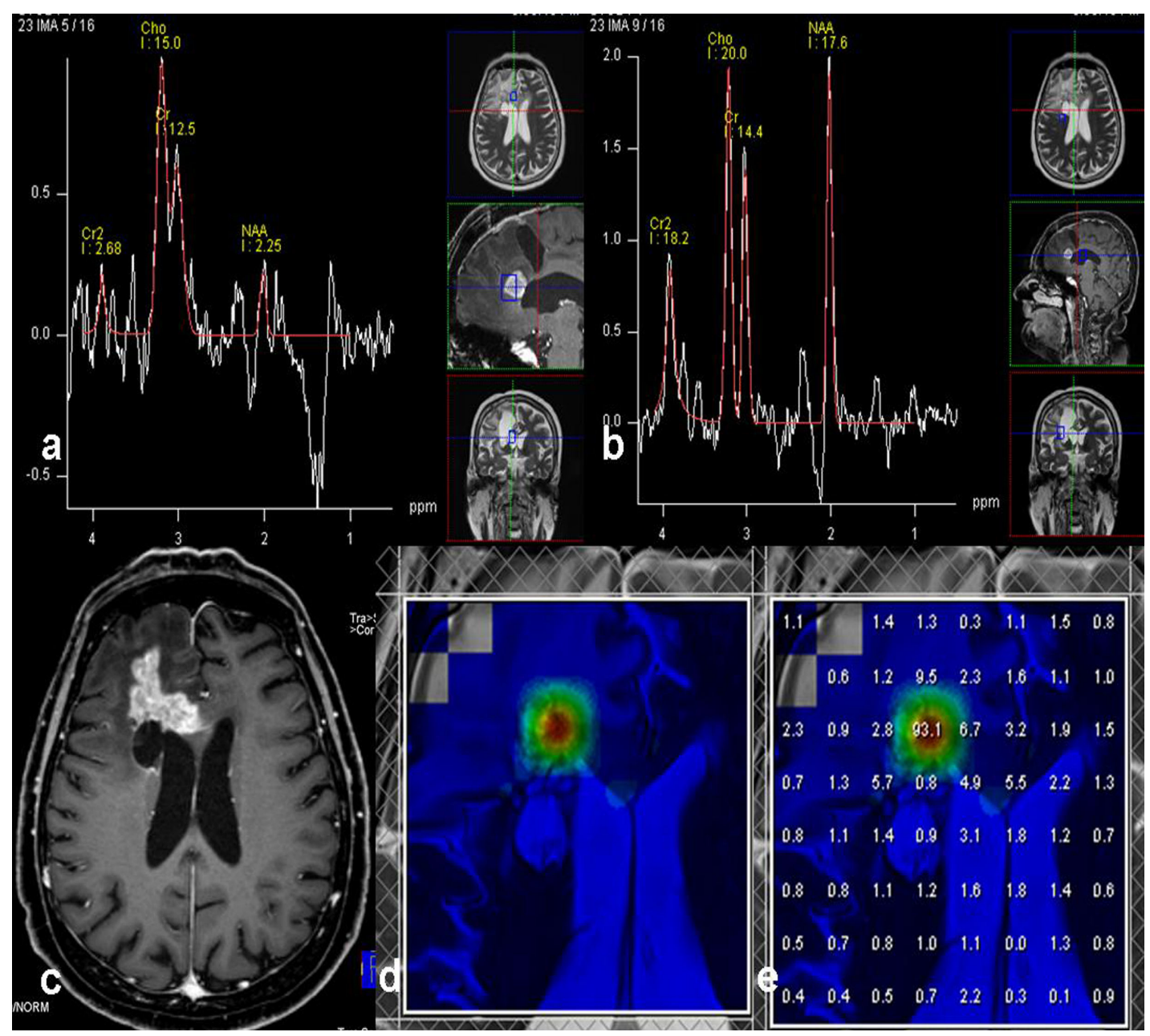

Figure 1. An example of MRS study on workstation: in MRS examination, with showing reference images and peak values of metabolites, we could differentiate tumour necrosis, recurrence and infiltration from each other. In high grade glioma case control after radiotherapy; a) High Cho/NAA ratios at the centre of lesion; b) decreased values at periphery c) contrast administered T1 axial images showed heterogeneous signal enhancement at post-radiotherapy area; d) Color map of metabolites and e) with superposed of peak metabolite information we showed there is only small malign transformation zone at the center of the area within the examined voxel of interest.

methods in use and with PRESS (point resolved spectroscopy) method signal-noise ratio is higher [2]. In STEAM (stimulated echo acquisition mode) method we could work with shorter TE values and we could get more detailed table of metabolites [3].

During examination, voxel of interest should not include especially scalp, sinuses, air or haemorrhagic foci. Normal CSF may dilute concentration but does not cause significant degradation of spectrum content. Also jewellery and ventriculoperitoneal shunts could disturb optimal settings of shimming and could change spectrum. Gadolinium could decrease the cho peak by \% 12 - 15, because of that MRS should be done before administration of contrast material. Because there is no certain normal international reference values for MRS metabolites, measurements could be compared with examined cases' own normal and abnormal regions. Also, some determined ratios of well known ratios can help in differential diagnosis as defined in Table 1 and Table 2.

On starting to examination, single or multi voxel protocol must be selected considering the purpose of the study. In searching of brain tumour which has got a heterogeneous or infiltrative pattern we should use multi-voxel protocol [4]. But in small lesions, posterior fossa tumors, or if there is a non removable metallic implant near the VOI, we should use single voxel protocol [1]. Especially for searching metabolic diseases, to achieve resonances of minor metabolites short TE values must be selected. Distribution of metabolites in resonance spectrum peaks could change due to: the device we use, the power of magnetic field. Also, shimming parameters 
can lead to different ppm values thorough the baseline but these changes are minor and do not cause significant diagnostic difficulties. In short TE examinations, Cr shows faster decay then other metabolites cause of that Cho/Cr ratio is higher at short examinations [5].

In grey matter NAA, Cr and glx are higher quantities, but in white matter Cho is relatively high [6] [7] Both hemispheres nearly have the same amount of metabolites but from occipital to frontal direction Cho decreases little bit. In basal ganglia, NAA and myo-inositol decrease. In pons, Cho and $\mathrm{Cr}$ are high but NAA and myo-inositol are low. In cerebellum, Cho and Cr are high but NAA is low [8].

\section{Metabolites}

NAA is the major neuronal metabolite and Cho, Cr, mIns, glutamate are the other ones. Whereas, the brain normally doesn't include high quantity of some metabolites (like lactate, hydroxy butyrate, acetone, glycine, succinate, pyruvate, alanine, lipid, phenylalanine, mannitol, ethanol, etc.) to definable on normal resonance line.

Diagnosis could be made by determining such abnormal metabolites or with determining changes of normal metabolites. Also with using a special technique, NAA's amid component's changes could be determined by administering histidine and homocarnosine. And so, brain $\mathrm{PH}$ levels could be measured by determination of NAA level changes. Also with whole brain spectroscopy, quantitative values of all metabolites could be measured.

Different components of metabolites could be useful for defining the different peak values of the same product. For example: NAA 2.02 and 2.6 ppm, Cr at 3.02 and 3.9 ppm, Glx between 2.05 - 2.4 and 3.65 - 3.8 ppm, glucose at 3.43 and $3.48 \mathrm{ppm}$, myo-inositol between 3.56 and 4.06. But, generally, only the established dominant ones to be considered for diagnosis.

Main metabolites: Cho (choline, $3.2 \mathrm{ppm}$ ), Cr (creatine, $3.0 \mathrm{ppm}$ ), NAA (n-acetile aspartic acid, $2.02 \mathrm{ppm}$ ), Mi (myo-inositole, 3.56 ppm) Lip (Lipid, 0.8 - 1.5 ppm), Lac ( lactate, 1.3 ppm) . The other metabolites will be explained later by shortly. Water peak visualised at $4.7 \mathrm{ppm}$ and it is used as reference value. All these metabolites that rises before water peak could be identified by spectral editing.

Cho: Marker of membrane proliferation, above normal values usually gives warning about malignity [9].

Cr: Peaks at $3.2 \mathrm{ppm}$. Useful as an internal reference [10].

NAA: Neuronal marker. The only situation causes increased NAA is Canavan disease. Generally all other diseases cause reduced NAA levels [11].

mI: As a glial proliferation marker, it increases in diabetes, Alzheimer disease, PMLE, Down syndrome; and decreases in stroke, tumours and encephalopathies. While $\mathrm{mI}$ is increases in Alzheimer and Down syndrome, it decreases in other cases of dementia [12] [13].

$\mathrm{mI}$ and glycine's peak values are so close to each other, they both resonate at 3.5 - 3.6 ppms (gly: 3.55-singlet, mIns: 3.52 - 3.61-multiplet). Myo-inositol makes multiplet at short TE's. It's strength decreases at long TE values. Contrary to this, glycine resonates as a singlet peak and its visualisation enhances at long TE values. Gly increases in stroke, encephalitis, Huntington, Parkinson, Alzheimer and Van der Knaap diseases [14].

In infantile periods, $\mathrm{mI}$ is the dominant peak at first months and Cho is also high at these times. But then mI's and Cho's peak values decreases as NAA, Cr increases. After the end of age 2, MRS patterns of metabolites becomes similar with adult level [15].

Table 1. Example of normal metabolite ratios for children and adults.

\begin{tabular}{ccccc}
\hline Normal & Neonatal & Infantile & Child & Adult \\
\hline NAA/Cho & $0.67 \pm 0.17$ & $1.56 \pm 0.67$ & $3.43 \pm 1.04$ & $2.37 \pm 0.40$ \\
Cho/Cr & $1.27 \pm 0.40$ & $0.84 \pm 0.21$ & $0.49 \pm 0.16$ & $1.05 \pm 0.23$ \\
\hline
\end{tabular}

Table 2. Example of metabolite ratios for differentiation of low and high grade glial tumors.

\begin{tabular}{ccll}
\hline Normal & $\mathrm{NAA} / \mathrm{Cr}=1.5 \pm 0.2$ & $\mathrm{Cho} / \mathrm{Cr}=1.05 \pm 0.23$ & $\mathrm{NAA} / \mathrm{Cho}=2.37 \pm 0.40$ \\
Gliom low grade & $\mathrm{NAA} / \mathrm{Cr}=1.12 \pm 0.48$ & $\mathrm{Cho} / \mathrm{Cr}=1.71 \pm 0.63$ & $\mathrm{NAA} / \mathrm{Cho}=0.65 \pm 0.37$ \\
Gliom high grade & $\mathrm{NAA} / \mathrm{Cr}=0.66 \pm 0.37$ & $\mathrm{Cho} / \mathrm{Cr}=1.95 \pm 0.72$ & $\mathrm{NAA} / \mathrm{Cho}=0.33 \pm 0.17$ \\
\hline
\end{tabular}


Glutamate/glutamine: Some times these couple of metabolites are called as Glx. Glx peaks mainly between 2.1 and 2.45. In MS plaques, hepatic encephalopathy, and Reyes syndrome, increased ammonia level causes increase in Glx peak values [16] [17].

GABA: It is visualised at 1.9, 2.3, 3.0 ppm's, but 2.3 ppm should be first choice because at 1.9 and 3.0 ppm values it overlaps by other dominant metabolites. Simply, for GABA 2.31 ppm and for Glutamine 2.62 pm values could be used. As a new method, MegaPRESS sequence could be used for quantitative differentiation of GABA [18].

Lactate (resonates at $1.3 \mathrm{ppm}$ ) is more prominent at longs TE values. In 1.5 tesla $150 \mathrm{~ms}$, in 3 tesla 140 ms is the most suitable values. Inversion of lactate at short TE values could be incomplete at 3 Tesla systems. But generally, in shorts TE's lactate is inverted but lipid is more prominent.

2-Hydroxyglutarate: gioma cases that have isocitrate dehydrogenase mutations. So, 2-hydroxyglutarate increases and visualised especially with TE: 90 - $100 \mathrm{~ms}$ at $2.25 \mathrm{ppm}$. Glioma cases that have high rate of this metabolite have good response to angiogenic therapy [19] [20].

Lipid-macromolecular peaks resonates at 0.8, 1.2 1.5, 6.0 ppms. $0.8-1.2$ ppm values usually called as lipid-macromolecular non-specific peak. If there is too much lipid we should use long TE for decrease lipids that have shorter TE values to get clear resonance graphics (It increases in tumours, metastasis and post-radiotherapy changes) [11] [21].

Pyruvate: it peaks at 2.37 ppm. Normally couldn't detected but increase and could be defined in pyruvate dehydrogenase enzyme deficiency [22].

Syllo-inositole: peaks at 3.35 ppm, increases in alcoholism and glioneuronal tumours. It can be rise in early infancy especially at examinations obtained in 3 Tesla systems. It can be superposed by taurine but taurine has simple peak while sylloinositole has a more complex one [23].

Guanidinoacetate: Increases at $3.78 \mathrm{ppm}$. It is lower in anaplastic astrocytomas then other gliomas [24].

Citrate: Increases at $2.6 \mathrm{ppm}$ in some brain tumours like PNET and high grade glial tumours [25].

Melanin: Peaks at $6.72 \mathrm{ppm}$. It can be detected in lesions that contain melanin.

Glucose: Peaks at 3.43 and 3.80 ppm. Increases in DM and hyperglycemia crisis.

Glutathione: Detected at 2.95 ppm. Increases in GBM, meningioma, and ischemic oxidative stress.

Additional to searching all these metabolites on resonance line, also there are some useful metabolite ratios to increase the strength of differential diagnosis (Table 1 and Table 2).

It's very hard to keep all those values defined on Table 1 and Table 2 in mind so we should check on tables when we need to. But with practical approach we can use these following simplified ratios especially when searching for gliomas.

NORMAL: NAA/Cr $>2.0$, NAA/Cho $>1.6$, Cho/Cr $<1.2$

ABNORMAL: NAA/Cr $<1.6$, NAA/Cho $<1.2, \mathrm{Cho} / \mathrm{Cr}>1.5$

Generally, instead of using MRS alone, we should use other conventional and multi parametric methods together for diagnosis. For example in high grade tumours; Cho and rCBV is high, in low grade tumours myo-inositol and glycine is high but rCBV is low. In PNET, Cho is high but NAA is low and taurine can also be high.

\subsection{Special Clues for Specific Diseases}

\subsubsection{Craniopharyngioma}

MRS establishes high levels of lipid and lactate due to rich cholesterol crystals and lipid deposits whereas normal neuronal metabolites are lower or absent. As cranial fossa base and bones are superposed and volume of hypophysis gland is lower than $2 \mathrm{~mm}$, MRS is not very effective. If craniopharyngioma is large enough, especially at 1.5 ppm, it could be seen that central lipid-cholesterol peaks are high but Cho-NAA peaks are not. However, high Cho/Cr levels and presence of NAA are detected in gliomas that mimic craniopharyngioma which are located at hypothalamic or chiasmatic region [26].

\subsubsection{Meningioma}

Cytosolic amino-acides like Alanine (1.48 ppm), Acetate (1.9 ppm), Valine (0.9 ppm), Leucine (3.6 ppm) could increase. Furthermore, increase in Glx peak between 2.1 - 2.4 ppm and Glutathione peak at 2.95 have been notified in some literature. With respect to these characteristics, it is possible to distinguish schwannomas located in the same region or atypically located meningiomas (intraosseous or intraventricular) [27]. 


\subsubsection{Etanol Intoxication}

At $1.2 \mathrm{ppm}$, an increased triplet peak may be defined.

\subsubsection{Ischemia}

In early phase of acute stroke NAA decreases and this decrease continues during the first week. Creatine acts similar to NAA. But choline levels are not reliable and they are variable. On the other hand, lactate level starts to increase a few minutes after stroke. It decreases again by reperfusion [1] [28] [29]. However although it is hard to detect at this level, penumbra that has normal NAA and high lactate levels may be detected by MRS and reperfusion can be provided and viability can be pursued [29].

\subsection{Hypoxic Ischemic Encephalopathy}

Lac and Glu increase and NAA ratio decreases. A severe variability in Cho levels is not expected.

\subsubsection{Abscess}

If it is tuberculosis abscess, lipid lactate levels increase, NAA and Cho decrease and a small increase is observed in aminoacid levels [30] [31].

In pyogenic abscess, increase of aminoacid levels are more apparent [30] [32].

In fungal abscess, lipid lactate levels increase more then aminoacid levels. Unlike Tbc abscess, especially in angioinvasive type, the intricated walls called crenelated outlines [30].

\subsubsection{Multiple Sclerosis}

NAA decreases, Cho, lactate and myoinositol increase [33] [34].

\subsubsection{Hepatic Encephalopathy}

Due to increase in ammonia level, Glu peak rises. Myoinositol and Cho decrease and lactate increases [35]. Decrease in Cho and Cr levels, particularly at follow-up imaging indicates poor prognosis [36]. According to the recent studies, decrease of myoinositol can be detected even at early phase. So it is possible to establish pathology during early phase and beginning of therapy can be expedited in suspected cases.

\subsubsection{Epilepsy}

Since the quality of spectrum is more clear and relatively more quantitative, MRS is implemented as single voxel. Multivoxel-CSI needs longer time, and since many of epilepsy patients are pediatric, they move during the process and distortion occurs in images. During acute phase, sodium/myoinositol pump increases myo-inositol levels. But the artefacts due to movements of epileptic ictus make it difficult to detect. In the interictal phase, NAA decreases due to neuronal decrement and myo-inositol shows moderate increases due to reactive astrogliosis in hypocampal sclerosis [1] [37]. In patients who take vigabatrin treatment, temporary T2 signal amplification may be seen in dentate nucleus, globus pallidus, brain stem and thalamus. In these regions, MRS is normal and is used especially to follow up signal differences due to vigabatrin. In epileptic cases MRS is used to follow up kethogenic diet. The recommended diet has low carbohydrate and high lipid [38]. The liver excrete ketoacides, kethon particules arising at $2 \mathrm{ppm}$, acetoasetate at 2.5 and $3.46 \mathrm{ppm}$. In epileptic cases, it is important to establish lateralisation of temporal lob which has normal signal appearance in conventional sequences but in fact generates epilepsy. NAA/Cr ratio decreases at abnormal site, but, as it is known in literature that contralateral side may show lower NAA/Cr level up to 50\%, comparing both sides must be done carefully [2] [4].

\subsection{Propylene Glycol}

A solvent which is used in several operations, several treatment protocols and in some drugs that are used in preoperative or intraoperative anesthesia, peaks at $1.13 \mathrm{ppm}$ at MRS. This solvent also exists in several drugs and benefical in security and follow-up of the treatment.

\subsubsection{RT Necrosis}

At 0 - 2 ppm interval, there is lip-macromolecule peak increase that supress other peaks occur [11] [39]. Beside this giant peak, signs of neuronal viability are low. 


\subsubsection{Psychiatric Cases}

During Lithium treatment, Cho levels diminishes [40]. Occasionally it is useful to decide whether the drug has been used or not. For other neuropsychiatric conditions, many data from literature show some complicated and inconsistent information that could not been summarised shortly in this paper.

\subsubsection{Kernicterus}

NAA/Cho ratio decreases, myo-inositol, taurin and Glx peaks arise [16]. Lactate level does not increase, so it is distinguishable from hypoxic ischemic conditions.

\subsubsection{Oligodendroglioma}

Tumour is identifiable as; myo-inositol is very high [41], Cho is very low, perfusion is highly increased and in enhancement is predominantly cortical.

\subsubsection{Lymphoma}

In MRS, lymphoma shows abnormally high lipid-lactate ratio, ADC levels are low relatively to other lesions. rCBV ratio is generally lower than expected $(<2.3)$. In metastasis and GBM these ratio is very high. In metastasis peritumoural perfusion has not increased versus to GBM. Discrimination of diagnosis is safe based on these indirect indicators. In cerebral lymphoma, very high increase of Cho is useful to distinguish from toxoplasma, tuberculosis and cryptococcus infection [42] [43].

\subsection{Metabolic Diseases}

In MSUD (maple syrupe urine disease): at 0.9 - $1.0 \mathrm{ppm}$ long branched-chained amino acids, in diabetic ketoacidosis at $2.2 \mathrm{ppm}$ acetone and at $3.4 \mathrm{ppm}$ glucose increase.

In galactosemia: galactitol (which mimics lactose) peak between 3.67 - 3.74 ppms.

In phenylketonuria: phenylalanine peak arises at $7.6 \mathrm{ppm}$.

In lipid storage diseases: high levels of lipid resonance occur generally at 0.7 - 1.6 ppms.

Increased lactate is obvious in mitochondria storage disease as Leigh, MERF (Myoclonus epilepsy with ragged-red fibers) and MELAS (Mitochondrial encephalomyopathy, lactic acidosis, and stroke-like episodes).

Scyllo-inositol increases at $3.35 \mathrm{ppm}$ in peroxisomal diseases.

For diagnosis and follow-up of glial tumours, especially perfusion MRI, all the imaging parameters should be used in collaboration. For example, low rCBV and high $\mathrm{ml} / \mathrm{Cr}$ indicates low grade tumour whereas $\mathrm{Cr}$ is low and it is at least grade III GBM. These alterations are proportional to Ki-67 which is a indicator of proliferation-mitosis and high Cho and low $\mathrm{Cr}$ are indicators of poor prognosis [44] [45]. Low grade tumours may transform to high grade tumours within 5 - 10 years. As tumour grade increases, myo-inositol levels decrease.

Tumours after radiotherapy, if rCBV $>1.3$ we have to think relapse instead of necrosis. If $\mathrm{rCBV}>1.75$, $\mathrm{Cho} / \mathrm{Cr}>1.83$ and $\mathrm{Cho} / \mathrm{NAA}>1.92$ it is acceptable as high grade. Peritumoural infiltration is possible if tissue which is surrounding lesions has $\mathrm{Cho} / \mathrm{Cr}$ ratio more than 1.3 times.

Cho may have misleading elevations in pilocytic astrositoma. This is due to increase of glycerophosphocholine instead of pathologic phosphocoline. Unfortunately discrimination is not possible with MRS. Clinical situations, age and conventional sequences may help. Beside these, in pilocytic astrositoma unassigned peak elevation occurs at $2.8 \mathrm{ppm}$ due to unsaturated fatty acids increase.

For differential diagnosis in such conditions, data from MRS should be used in conjunction with conventional and advanced neuroradiological findings. For example, in T2 perfusion, signal fall to $75 \%$ level is important, as line approaches to baseline at normal brain and gliomas. In lymphoma and pilocytic astrocytoma, it may arise to higher than baseline. But in meningiomas it is remote from baseline. Unlike choroid plexus papillomas, intraventricular meningiomas has rich perfusion. In choroid plexus papillomas myo-inositol is very high. As atypia and malignity progress, myo-inositol falls and Cho increases [44]. Though to looking intricated, we radiologists must be used to combine these rules in daily practice to solve the complex imaging findings.

As another example, in addition to MRS, ADC (apparent diffusion coefficient) values gain advantage when making diagnosis. At posterior fossa tumours, which display similar conventional findings, ADC measurement can help to differentiation. Because, ADC value is between $1.0-1.3\left(\times 10^{-3} \mathrm{~mm}^{2} / \mathrm{s}\right)$ for ependymoma, lower than 0.7 for medulloblastoma, and generally higher than 1.4 for pilocytic astrocytoma. 


\section{Conclusion}

In conclusion, with this condensed review, despite a high technical-physical burden about MRS which is highly complicated and intense, we provide much practical information. We also believe to ensure knowing basic informations from performing protocol to permutation of metabolites as well as essential data about rare metabolites and differences on spectrum made by several diseases. With development of software and hardware, utilisation of MRS in diagnostic radiology will expand beyond today.

\section{References}

[1] Oz, G., Alger, J.R., Barker, P.B., Bartha, R. and Bizzi, A. (2014) MRS Consensus Group. Clinical Proton MR Spectroscopy in Central Nervous system Disorders. Radiology, 270, 658-679. http://dx.doi.org/10.1148/radiol.13130531

[2] Burtscher, I.M. and Holtas, S. (2001) Proton MR Spectroscopy in Clinical Routine. Journal of Magnetic Resonance Imaging, 13, 560-567. http://dx.doi.org/10.1002/jmri.1079

[3] Frahm, J., Merboldt, K.D. and Hänicke, W. (1969) Localised Proton Spectroscopy Using Stimulated Echoes. Journal of Magnetic Resonance, 72, 502-508.

[4] Gillard, J.H., Waldman, A.D. and Barker, P.B. (2005) Clinical MR Neuroimaging. Cambridge University Press, New York.

[5] Zimmerman, R.A. and Wang, Z.J. (1997) The Value of Proton MR Spectroscopy in Pediatric Metabolic Brain Disease. American Journal of Neuroradiology, 18, 1872-1879.

[6] Kreis, R., Ernst, T. and Ross, B.D. (1993) Absolute Quantitation of Water and Metabolites in the Human Brain. II, Metabolite Concentrations. Journal of Magnetic Resonance, Series B, 102, 9-19. http://dx.doi.org/10.1006/jmrb.1993.1056

[7] Doyle, T.J., Bedell, B.J. and Narayana, P. (1995) Relative Concentrations of Proton MR Visible Neurochemicals in Gray and White Matter in Human Brain. Magnetic Resonance in Medicine, 33, 755-759. http://dx.doi.org/10.1002/mrm.1910330603

[8] Soher, B.J., van Zijl, P., Duyn, J.H. and Barker, P.B. (1996) Quantitative Proton MR Spectroscopic Imaging of the Human Brain. Magnetic Resonance in Medicine, 35, 356-363. http://dx.doi.org/10.1002/mrm.1910350313

[9] Maheshwari, S.R., Fatterpekar, G.M., Castillo, M. and Mukherji, S.K. (2000) Proton MR Spectroscopy of the Brain. Seminars in Ultrasound, CT and MRI, 21, 434-451. http://dx.doi.org/10.1016/S0887-2171(00)90036-2

[10] Danielsen, E.R., Ross, B. and Danielsen, E.R. (1999) Magnetic Resonance Spectroscopy Diagnosis of Neurological Diseases. Marcel Dekker, New York, 30-34.

[11] Castillo, M., Kwock, L., Scatliff, J. and Mukherji, S.K. (1998) Proton MR Spectroscopy in Neoplastic and Non-Neoplastic Brain Disorders. Magnetic Resonance Imaging Clinics of North America, 6, 1-20.

[12] Miller, B.L., Moats, R.A., Shonk, T., Ernst, T., Woolley, S. and Ross, B.D. (1993) Alzheimer Disease: Depiction of Increased Cerebral Myo-Inositol with Proton MR Spectroscopy. Radiology, 187, 433-437. http://dx.doi.org/10.1148/radiology.187.2.8475286

[13] Ernst, T., Chang, L., Melchor, R. and Mehringer, C.M. (1997) Frontotemporal Dementia and Early Alzheimer Disease: Differentiation with Frontal Lobe H-1 MR Spectroscopy. Radiology, 203, 829-836. http://dx.doi.org/10.1148/radiology.203.3.9169712

[14] Terek, D., Koroglu, O.A., Gunes, S., Yalaz, M., Akisu, M., Uçar, S.K., Gokben, S., Coker, M. and Kultursay, N. (2012) Diagnostic Tools of Metabolic and Structural Brain Disturbances in Neonatal Non-Ketotic Hyperglycinemia. Pediatrics International, 54, 717-720. http://dx.doi.org/10.1111/j.1442-200X.2012.03591.X

[15] Wozniak, J.R. and Lim, K.O. (2006) Advances in White Matter Imaging: A Review of in Vivo Magnetic Resonance Methodologies and Their Applicability to the Study of Development and Aging. Neuroscience \& Biobehavioral Reviews, 30, 762-774. http://dx.doi.org/10.1016/j.neubiorev.2006.06.003

[16] Córdoba, J., Sanpedro, F., Alonso, J. and Rovira, A. (2002) 1H Magnetic Resonance in the Study of Hepatic Encephalopathy in Humans. Metabolic Brain Disease, 17, 415-429. http://dx.doi.org/10.1023/A:1021926405944

[17] Novotny, E.J., Fulbright, R.K., Pearl, P.L., Gibson, K.M. and Rothman, D.L. (2003) Magnetic Resonance Spectroscopy of Neurotransmitters in Human Brain. Annals of Neurology, 54, 25-31. http://dx.doi.org/10.1002/ana.10697

[18] O’Gorman, R.L., Michels, L., Edden, R.A., Murdoch, J.B. and Martin, E. (2011) In Vivo Detection of GABA and Glutamate with MEGA-PRESS: Reproducibility and Gender Effect. Journal of Magnetic Resonance Imaging, 33, 12621267. http://dx.doi.org/10.1002/jmri.22520

[19] Lazovic, J., Soto, H., Piccioni, D., Ru, J.R.L., Li, S., Mirsadraei, L. and Pop, B.W. (2012) Detection of 2-Hydroxyglutaric 
Acid in Vivo by Proton Magnetic Resonance Spectroscopy in U87 Glioma Cells Overexpressing Isocitrate Dehydrogenase-1 Mutation. Neuro-Oncology, 14, 1465-1472.

[20] Elkhaled, A., Jalbert, L.E., Phillips, J.J., Yoshihara, H.A., Parvataneni, R., Srinivasan, R. and Nelson, S.J. (2012) Magnetic Resonance of 2-Hydroxyglutarate in IDH1-Mutated Low-Grade Gliomas. Science Translational Medicine, 4, $116 r a 5$.

[21] Kaminogo, M., Ishimaru, H., Morikawa, M., Ochi, M., Ushijima, R., Tani, M. and Shibata, S. (2001) Diagnostic Potential of Short Echo Time MR Spectroscopy of Gliomas with Single-Voxel and Point-Resolved Spatially Localized Proton Spectroscopy of Brain. Neuroradiology, 43, 353-363. http://dx.doi.org/10.1007/s002340000473

[22] Zand, D.J., Simon, E.M., Pulitzer, S.B., Wang, D.J., Wang, Z.J., Rorke, L.B., Palmieri, M. and Berry, G.T. (2003) In Vivo Pyruvate Detected by MR Spectroscopy in Neonatal Pyruvate Dehydrogenase Deficiency. American Journal of Neuroradiology, 24, 1471-1474.

[23] Rae, C.D. (2014) A Guide to the Metabolic Pathways and Function of Metabolites Observed in Human Brain 1H Magnetic Resonance Spectra. Neurochemical Research, 39, 1-36. http://dx.doi.org/10.1007/s11064-013-1199-5

[24] Panigrahy, A. and Blüml, S. (2009) Neuroimaging of Pediatric Brain Tumors: From Basic to Advanced Magnetic Resonance Imaging. Child Neurology, 24, 1343-1365. http://dx.doi.org/10.1177/0883073809342129

[25] Blüml, S., Panigrahy, A., Laskov, M., Dhall, G., Krieger, M.D., Nelson, M.D. and Gilles, F.H. (2011) Elevated Citrate in Pediatric Astrocytomas with Malignant Progression. Neuro-Oncology, 13, 1107-1117. http://dx.doi.org/10.1093/neuonc/nor087

[26] Mohammad, F.F., Hasan, D.I. and Ammar, M.G. (2014) MR Spectroscopy and Diffusion MR Imaging in Characterisation of Common Sellar and Supra-Sellar Neoplastic Lesions. The Egyptian Journal of Radiology and Nuclear Medicine, 45, 859-867. http://dx.doi.org/10.1016/j.ejrnm.2014.04.012

[27] Yue, Q., Isobe, T., Shibata, Y., Anno, I., Kawamura, H., Yamamoto, Y. and Matsumura, A. (2008) New Observations Concerning the Interpretation of Magnetic Resonance Spectroscopy of Meningioma. European Radiology, 18, 29012911. http://dx.doi.org/10.1007/s00330-008-1079-6

[28] Castillo, M., Kwock, L. and Mukherji, S.K. (1996) Clinical Applications of Proton MR Spectroscopy. American Journal of Neuroradiology, 17, 1-15.

[29] Gillard, J.H., Waldman, A.D. and Barker, P.B. (2005) Clinical MR Neuroimaging. Cambridge University Press, New York.

[30] Luthra, G., Parihar, A., Nath, K., Jaiswal, S., Prasad, K.N., Husain, N. and Gupta, R.K. (2007) Comparative Evaluation of Fungal, Tubercular, and Pyogenic Brain Abscesses with Conventional and Diffusion MR Imaging and Proton MR Spectroscopy. American Journal of Neuroradiology, 28, 1332-1338. http://dx.doi.org/10.3174/ajnr.A0548

[31] Cecil, K.M. and Lenkinski, R.E. (1998) Proton MR Spectroscopy in Inflammatory and Infectious Brain Disorders. Neuroimaging Clinics of North America, 8, 863-880.

[32] Lai, P.H., Li, K.T., Hsu, S.S., Hsiao, C.C., Yip, C.W., Ding, S. and Pan, H.B. (2005) Pyogenic Brain Abscess: Findings from in Vivo 1.5-T and 11.7-T in Vitro Proton MR Spectroscopy. American Journal of Neuroradiology, 26, 279288.

[33] Narayana, P.A. (2005) Magnetic Resonance Spectroscopy in the Monitoring of Multiple Sclerosis. Journal of Neuroimaging, 15, 46-57. http://dx.doi.org/10.1177/1051228405284200

[34] Narayana, P.A., Doyle, T.J., Lai, D. and Wolinsky, J.S. (1998) Serial Proton Magnetic Resonance Spectroscopic Imaging, Contrast-Enhanced Magnetic Resonance Imaging, and Quantitative Lesion Volumetry in Multiple Sclerosis. Annals of Neurology, 43, 56-57. http://dx.doi.org/10.1002/ana.410430112

[35] Savranlar, A., Ozer, T., Aydemir, S., Alkan, A. and Borazan, A. (2005) Proton Magnetic Resonance Spectroscopy Findings of Wilson's Disease Case Report. Journal of Neurological Sciences (Turkish), 22, 297-303.

[36] Saksena, S., Rai, V., Saraswat, V.A., Rathore, R.S., Purwar, A., Kumar, M. and Gupta, R.K. (2008) Cerebral Diffusion Tensor Imaging and in Vivo Proton Magnetic Resonance Spectroscopy in Patients with Fulminant Hepatic Failure. Journal of Gastroenterology and Hepatology, 23, 111-119. http://dx.doi.org/10.1111/j.1440-1746.2007.05158.X

[37] Brand, A., Richter-Landsberg, C. and Leibfritz, D. (1993) Multinuclear NMR Studies on the Energy Metabolism of Glial and Neuronal Cells. Developmental Neuroscience, 15, 289-298. http://dx.doi.org/10.1159/000111347

[38] Stafstrom, C.E. (2004) Dietary Approaches to Epilepsy Treatment: Old and New Options on the Men. Epilepsy Currents, 4, 215-222. http://dx.doi.org/10.1111/j.1535-7597.2004.46001.x

[39] Rock, J.P., Hearshen, D., Scarpace, L., Croteau, D., Gutierrez, J., Fisher, J.L. and Mikkelsen, T. (2002) Correlations between Magnetic Resonance Spectroscopy and Image-Guided Histopathology, with Special Attention to Radiation Necrosis. Neurosurgery, 51, 912-920.

[40] Davanzo, P., Thomas, M.A., Yue, K., Oshiro, T., Belin, T., Strober, M. and McCracken, J. (2001) Decreased Anterior 
Cingulate Myo-Inositol/Creatine Spectroscopy Resonance with Lithium Treatment in Children with Bipolar Disorder. Neuropsychopharmacology, 24, 359-369. http://dx.doi.org/10.1016/S0893-133X(00)00207-4

[41] Kleihues, P. and Cavenee, W.K. (2000) Pathology and Genetics of Tumours of the Nervous System. International Agency for Research on Cancer, Lyon.

[42] Chinn, R.J., Wilkinson, I.D., Hall-Craggs, M.A., Paley, M.N., Miller, R.F., Kendall, B.E. and Harrison, M.J. (1995) Toxoplasmosis and Primary Central Nervous System Lymphoma in HIV Infection: Diagnosis with MR Spectroscopy. Radiology, 197, 649-654. http://dx.doi.org/10.1148/radiology.197.3.7480733

[43] Schultz Jr., K.D., Petronio, J., Narad, C. and Hunter, S.B. (1997) Solitary Intracerebral Juvenile Xanthogranuloma. Pediatric Neurosurgery, 26, 315-321. http://dx.doi.org/10.1159/000121212

[44] Howe, F.A., Barton, S.J., Cudlip, S.A., Stubbs, M., Saunders, D.E., Murphy, M. and Griffiths, J.R. (2003) Metabolic Profiles of Human Brain Tumors Using Quantitative in Vivo 1H Magnetic Resonance Spectroscopy. Magnetic Resonance in Medicine, 49, 223-232. http://dx.doi.org/10.1002/mrm.10367

[45] Furuya, S., Naruse, S., Ide, M., Morishita, H.E., Kizu, O., Ueda, S. and Maeda, T. (1997) Evaluation of Metabolic Heterogeneity in Brain Tumors Using 1H-Chemical Shift Imaging Method. NMR in Biomedicine, 10, 25-30. http://dx.doi.org/10.1002/(SICI)1099-1492(199701)10:1<25::AID-NBM445>3.0.CO;2-M 
Scientific Research Publishing (SCIRP) is one of the largest Open Access journal publishers. It is currently publishing more than 200 open access, online, peer-reviewed journals covering a wide range of academic disciplines. SCIRP serves the worldwide academic communities and contributes to the progress and application of science with its publication.

Other selected journals from SCIRP are listed as below. Submit your manuscript to us via either submit@scirp.org or Online Submission Portal.
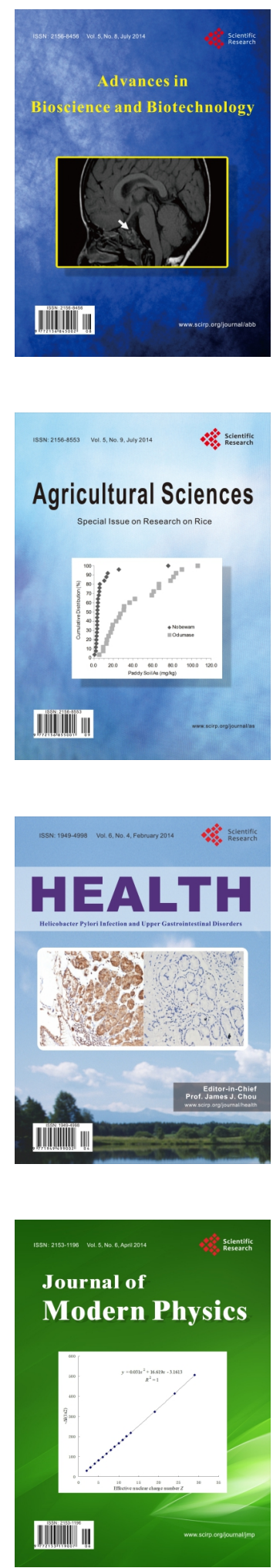
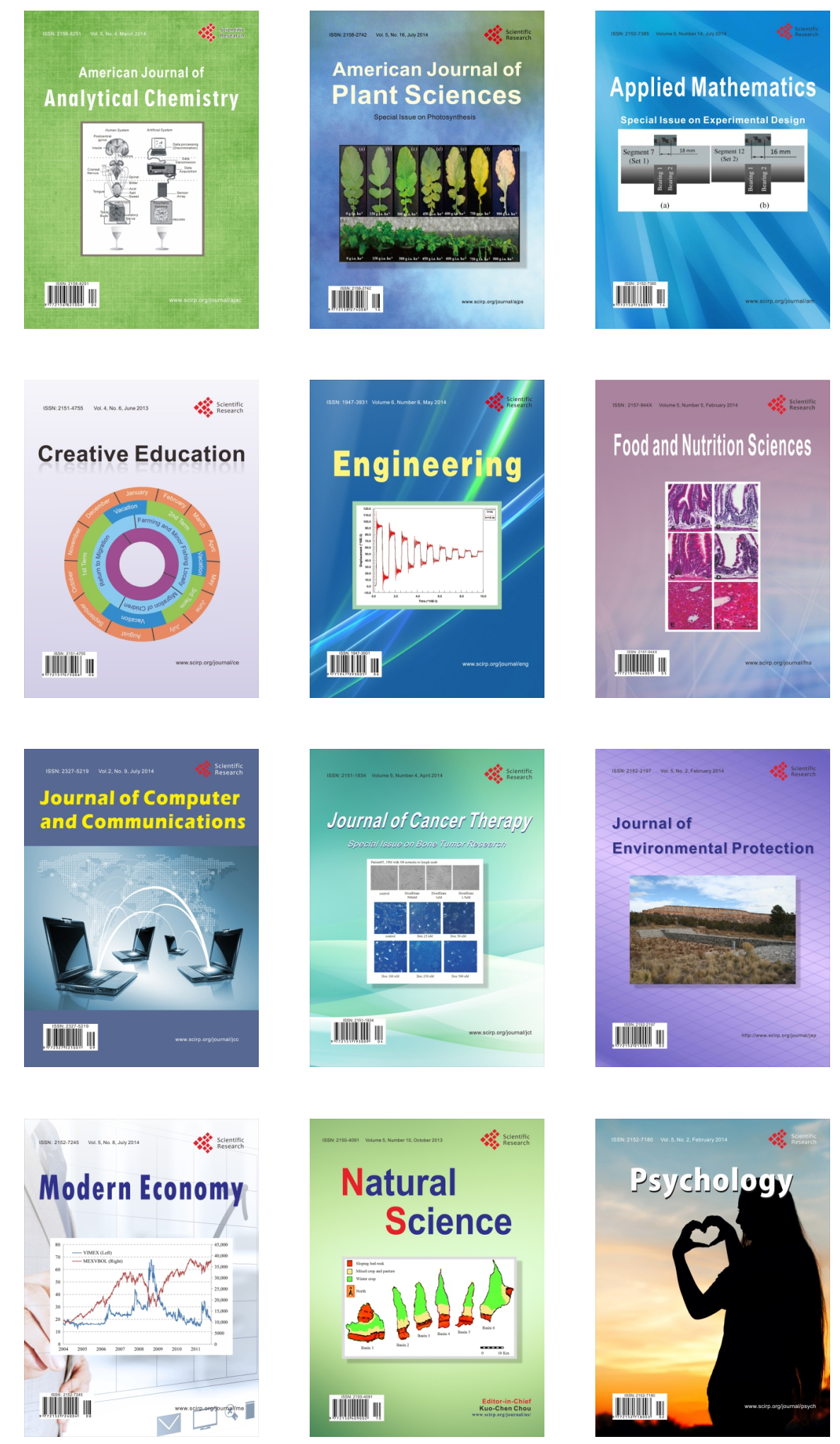